\title{
MODELAGEM MULTICRITÉRIO PARA SELEÇÃO DE INTERVALOS DE MANUTENÇÃO PREVENTIVA BASEADA NA TEORIA DA UTILIDADE MULTIATRIBUTO
}

\author{
Adiel Teixeira de Almeida \\ Programa de Pós-Graduação em Engenharia de \\ Produção (PPGEP) \\ Universidade Federal de Pernambuco (UFPE) \\ Recife - PE \\ aalmeida@ufpe.br
}

Recebido em 01/2004; aceito em 11/2004

Received January 2004; accepted November 2004

\section{Resumo}

O problema de seleção de intervalos ou periodicidade para manutenção preventiva foi analisado através de um modelo de decisão multicritério. Este modelo de decisão apóia decisores na escolha da melhor combinação das conseqüências custo e confiabilidade, analisando um contexto de sistema de produção de serviços. O modelo de decisão é baseado na Teoria da Utilidade Multiatributo (MAUT). O artigo apresenta os principais aspectos teóricos e os elementos básicos utilizados para construção do modelo. Uma aplicação numérica é apresentada para ilustrar o uso do modelo de decisão.

Palavras-chave: manutenção preventiva; apoio a decisão multicritério; teoria da utilidade multiatributo (MAUT).

\begin{abstract}
The problem of interval selection for preventive maintenance has been analysed through a multicriteria decision model. This decision model supports decision makers in choosing the best combination of consequences cost and reliability, analysing a context of service production systems. The decision model is based on the multiattribute utility theory (MAUT). The paper presents the main theoretical and practical aspects related to model building. A numerical application is presented in order to illustrate the use of the decision model.
\end{abstract}

Keywords: preventive maintenance; multicriteria decision support; multiattribute utility theory. 


\section{Introdução}

Numa análise da literatura se observa que há pouco uso de métodos multicritério em problemas de manutenção, especialmente para modelar as preferências dos decisores.

Particularmente em problemas de manutenção preventiva muitos artigos apresentam modelos de decisão sem abordagem multicritério (Barlow \& Hunter, 1960; Glasser, 1969; Dekker \& Scarf, 1998; Percy \& Kobbacy, 2000). Em contraste, relativamente pouco trabalho tem sido apresentado na literatura com AMD (Kralja \& Petrovicb, 1995; Chareonsuk et al., 1997; Gopalaswamy et al., 1993). Pode-se observar que os problemas de manutenção preventiva no contexto de manufatura ou produção de bens não necessitam de uma abordagem multicritério. Entretanto, no contexto de produção de serviços, a abordagem multicritério muitas vezes é essencial.

Este artigo apresenta um modelo de decisão multicritério para manutenção preventiva baseado em teria da utilidade multiatributo (MAUT - multi-attribute utility theory). As preferências do decisor são modeladas com o objetivo de representar os múltiplos objetivos relativos ao problema de manutenção preventiva. O artigo apresenta o modelo e seus fundamentos teóricos, incluindo também uma aplicação numérica. Esta aplicação é baseada num estudo real conduzido em uma empresa de energia elétrica.

\section{O Problema}

A seguir têm-se os principais aspectos do problema de manutenção preventiva, incluindo a estrutura básica e o contexto do problema. Foi considerado que este problema está relacionado a um equipamento, um componente ou um sistema. Assim, quando o texto se refere a um sistema, pode-se considerar qualquer um destes casos, não havendo diferença na modelagem desenvolvida.

Os principais elementos a serem analisados são: espaço de ações, estado da natureza, e conseqüências. As conseqüências são associadas aos objetivos, conforme as abordagens de MAUT e de teoria da decisão (Berger, 1985; Raiffa, 1970; Keeney \& Raiffa, 1976).

O estado da natureza $\theta$ corresponde ao parâmetro ou parâmetros da função probabilística da confiabilidade do sistema, representando o comportamento deste sistema. Esta função pode ser uma distribuição de Weibull, usualmente encontrada como um bom ajuste para dados de falhas de sistemas que se deterioram com o tempo (O'Connor, 1985). O parâmetro $\theta$ pode ser: conhecido ou desconhecido. No último caso, considera-se que o conhecimento a priori de especialistas sobre as incertezas relacionadas ao $\theta$ são representadas por uma distribuição de probabilidade $\pi(\theta)$. Uma abordagem de confiabilidade Bayesiana tem sido freqüentemente aplicada nestes casos (Martz \& Waller, 1982).

O espaço de ações corresponde ao conjunto de alternativas disponível para o decisor. Para o problema analisado neste artigo, o espaço de ações é discreto, sendo cada alternativa $\mathrm{a}_{\mathbf{i}}$ correspondente ao possível intervalo de tempo de manutenção preventiva $t_{p}$. Valores de $t_{p}$, geralmente a intervalos pré-definidos, são estabelecidos dentro das necessidades de um contexto gerencial, tais como: mensal, quinzenal, semanal, diário.

A cada alternativa $t_{p}$ deste conjunto há uma correspondente consequiência que é uma combinação do custo e do desempenho no funcionamento do sistema. Este desempenho neste 
tipo de problema é usualmente representado pela confiabilidade do sistema (Glasser, 1969; O'Connor, 1985). Assim, as conseqüências a serem obtidas são: custo C e confiabilidade R. Estas conseqüências estão relacionadas aos objetivos do decisor que esteja à frente do problema de determinação do intervalo adequado para manutenção preventiva. Estes objetivos dependem de vários aspectos relacionados ao contexto do problema. Os aspectos mais importantes estão associados ao tipo de sistema de produção. Dois tipos são considerados de interesse para este problema: sistema de produção de bens (manufatura) e sistema de produção de serviço.

Em sistemas de produção de manufatura, os modelos de decisão geralmente não precisam de uma modelagem multicritério. Neste tipo de sistema o cliente não está diretamente em contato com o sistema de produção, quando este cliente está sendo servido. Isto é, o produto pode ser recebido pelo cliente sem distúrbios causado por falhas no sistema de produção. Na maioria dos casos, as perdas devido às falhas ou interrupção por manutenção preventiva podem ser contabilizadas de forma monetária. Isto significa que as consequiências de falhas são transformada em custos. Assim, os objetivos são atendidos reduzindo uma função de custo final.

Em sistemas de produção de serviços, a abordagem multicritério é necessário, em geral. Neste sistema o cliente está em direto contato com o sistema de produção. O produto é produzido enquanto o cliente está sendo servido. Neste caso, o produto recebido pelo cliente é afetado com os distúrbios decorrentes de falhas no sistema de produção. Assim, as perdas decorrentes de falhas ou interrupções por manutenção preventiva não podem ser contabilizadas simplesmente em forma monetária. As consequiências de interrupção no serviço podem afetar o futuro desejo de contratação do cliente por aquele fornecedor, ou mesmo levar a cancelar o contrato presente. Neste caso, as conseqüências de falhas não podem ser transformadas em custos. Os objetivos neste sistema são atendidos tentando reduzir custos em combinação com outros objetivos, tais como: disponibilidade, confiabilidade do sistema de produção, tempo de interrupção do sistema, e qualidade do serviço.

Portanto, para sistemas de produção de serviços um modelo multicritério é mais apropriado, para o propósito de combinar os múltiplos objetivos a serem atendidos. O problema analisado neste artigo está tratando de duas conseqüências de interesse para o decisor: o custo $C_{i}$ e a confiabilidade $R_{i}$, relacionadas à alternativa $a_{i}$.

\section{O Modelo de Decisão}

O modelo de decisão para tratar o problema de manutenção preventiva descrito acima é apresentado a seguir.

Conforme abordagem de MAUT (Keeney \& Raiffa, 1976; Gomes et al., 2002), as preferências sobre as consequiências são modeladas de modo a fornecer a função utilidade multiatributo. Esta função incorpora a estrutura de preferência do decisor em relação às consequiências: custo e confiabilidade. Sejam o custo $\mathrm{C}$ e a confiabilidade $\mathrm{R}$, relativos à alternativa $\mathrm{a}_{\mathrm{i}}$ representados por $\mathrm{C}_{\mathrm{i}}$ e $\mathrm{R}_{\mathrm{i}}$, respectivamente.

As preferências são modeladas em duas etapas. A primeira modelagem é aplicada sobre $\mathrm{C}$ e $\mathrm{R}$, a fim de obter as funções utilidade unidimensionais relativas a $\mathrm{C}$ e $\mathrm{R}$, respectivamente, U(C) e U(R). Em seguida é aplicada a segunda etapa a fim de obter a função utilidade multiatributo $\mathrm{U}(\mathrm{C}, \mathrm{R})$. 
Dado o estado da natureza $\theta$ e a alternativa $\mathrm{a}_{\mathrm{i}}$, a utilidade $\mathrm{U}\left(\theta, \mathrm{a}_{\mathrm{i}}\right)$ deverá ser encontrada, representando os valores obtidos pelo decisor no caso de cada alternativa $a_{i}$ para um dado estado da natureza $\theta$. Se os parâmetros relativos ao estado da natureza $\theta$ são conhecidos, então o decisor, quer escolher a alternativa $a_{i}$ para a qual a função utilidade $U\left(\theta, a_{i}\right)$ tem máximo valor.

$\mathrm{Na}$ abordagem de teoria da decisão Bayesiana, associada com MAUT (Berger, 1985), a probabilidade a priori $\pi(\theta)$ é levada em consideração. Assim, o decisor procura maximizar a utilidade esperada $\mathrm{E}_{\theta} \mathrm{U}\left(\theta, \mathrm{a}_{\mathrm{j}}\right)$, dada por

$$
\mathrm{E}_{\theta} \mathrm{U}\left(\mathrm{a}_{\mathrm{i}}, \theta\right)=\int_{\theta} \mathrm{U}\left(\mathrm{a}_{\mathrm{i}}, \theta\right) \pi(\theta)
$$

O problema é resolvido conforme (2), ou seja, escolhendo-se a alternativa $a_{i}$ tal que a utilidade esperada $\mathrm{E}_{\theta} \mathrm{U}\left(\theta, \mathrm{a}_{\mathrm{i}}\right)$ seja maximizada.

$$
\underset{\mathrm{a}_{\mathrm{i}}}{\operatorname{Max}} \int_{\theta} \mathrm{U}\left(\mathrm{a}_{\mathrm{i}}, \theta\right) \pi(\theta)
$$

Para se encontrar a utilidade $U\left(a_{i}, \theta\right)$, a função utilidade sobre as conseqüências, denotada por $\mathrm{U}(\mathrm{C}, \mathrm{R})$ é considerada. A utilidade $\mathrm{U}\left(\mathrm{a}_{\mathrm{i}}, \theta\right)$ corresponde à utilidade do estado da natureza $\theta \mathrm{e}$ alternativa $a_{i} \cdot U(C, R)$ está relacionada diretamente com o valor das conseqüências no espaço de consequiências $(C, R)$. Esta função é obtida através do processo de modelagem de preferências junto ao decisor. A utilidade $U\left(a_{i}, \theta\right)$ é obtida com base na função utilidade $\mathrm{U}(\mathrm{C}, \mathrm{R})$, aplicando a propriedade de linearidade da teoria da utilidade (Berger, 1985; Almeida \& Souza, 1993), donde se tem que $\mathrm{U}\left(\mathrm{a}_{\mathrm{i}}, \theta\right)$ é a o valor esperado da utilidade $\mathrm{U}(\mathrm{C}, \mathrm{R})$ :

$$
\mathrm{U}\left(\mathrm{a}_{\mathrm{i}}, \theta\right)=\int_{\mathrm{R}} \int_{\mathrm{C}} \mathrm{U}(\mathrm{C}, \mathrm{R}) \operatorname{Pr}\left(\mathrm{C}, \mathrm{R} \mid \mathrm{a}_{\mathrm{i}}, \theta\right) \mathrm{dCdR}
$$

\subsection{Função confiabilidade}

Dada a função densidade de probabilidade $f(t)$ para a o tempo de sobrevivência $t$, uma função confiabilidade $\mathrm{R}(\mathrm{t})$ pode ser obtida (O'Connor, 1985). A confiabilidade $\mathrm{R}=\mathrm{R}\left(\mathrm{t}_{\mathrm{p}}\right.$ ) corresponde a probabilidade de que o tempo de sobrevivência t será maior do que o tempo $\mathrm{t}_{\mathrm{p}}$. Assim, a função confiabilidade $\mathrm{R}=\mathrm{R}\left(\mathrm{t}_{\mathrm{p}}\right)$, é dada por:

$$
R=R\left(t_{p}\right)=\operatorname{Pr}\left[t>t_{p}\right]=\int_{t_{p}}^{\infty} f(t) d t
$$

\subsection{Função custo}

Vários modelos (Barlow \& Hunter, 1960; Glasser, 1969; Chareonsuk et al., 1997) são apresentados para a função custo, neste tipo de problema, donde procura-se minimizar a função c(t), dada por: 


$$
\mathrm{c}(\mathrm{t})=\mathrm{C}(\mathrm{t}) / \mathrm{T}(\mathrm{t})
$$

onde,

$$
\begin{aligned}
& \mathrm{C}(\mathrm{t})=\text { custo esperado por ciclo, } \\
& \mathrm{T}(\mathrm{t})=\text { tempo de ciclo esperado. }
\end{aligned}
$$

Dado o intervalo de manutenção preventiva $t_{p}, T\left(t_{p}\right)$ é obtido com segue:

$$
T\left(t_{p}\right)=u_{f}+t_{p}\left[1-F\left(t_{p}\right)\right]
$$

Onde, $\mathrm{u}_{\mathrm{f}}$ é o tempo de vida esperado, dado o intervalo $0 \mathrm{a} \mathrm{t}_{\mathrm{p}} \cdot \mathrm{F}\left(\mathrm{t}_{\mathrm{p}}\right)$ é a função distribuição de probabilidade para o tempo de sobrevivência, considerando $t=t_{p}$. Ou seja, dada a função densidade de probabilidade $\mathrm{f}(\mathrm{t})$ para o tempo de sobrevivência t, tem-se:

$$
F\left(t_{p}\right)=\int_{0}^{t_{p}} f(t) d t
$$

Por conseguinte:

$$
1-F\left(t_{p}\right)=\int_{t_{p}}^{\infty} f(t) d t
$$

Assim:

$$
T\left(t_{p}\right)=\int_{0}^{t_{p}} t f(t) d t+t_{p} \int_{t_{p}}^{\infty} f(t) d t
$$

Dado $\mathrm{C}_{\mathrm{f}}$ como o custo de manutenção por falha unitário e $\mathrm{C}_{\mathrm{p}}$ como o custo de manutenção preventiva unitário, então o custo esperado por ciclo pode ser obtido para $\mathrm{t}_{\mathrm{p}}$.

$$
\begin{aligned}
& C\left(t_{p}\right)=C_{f} F\left(t_{p}\right)+C_{p}\left[1-F\left(t_{p}\right)\right] \\
& C\left(t_{p}\right)=C_{f} \int_{0}^{t_{p}} f(t) d t+C_{p} \int_{t_{p}}^{\infty} f(t) d t
\end{aligned}
$$

Aplicando (4), (6) e (7) em (5), tem-se:

$$
C=c\left(t_{p}\right)=\frac{C_{f}(1-R)+C_{p} R}{t_{p} R+\int_{0}^{t_{p}} t f(t) d t}
$$

Conforme mencionado previamente, vários modelos estão concentrados na minimização da função em (8). Então a solução ótima para (8) é obtida tomando-se a derivada com respeito a $\mathrm{t}_{\mathrm{p}}$, solucionando quando se iguala a zero. Entretanto, para várias funções de probabilidade $\mathrm{f}(\mathrm{t})$, tal qual a Weibull, não é possível obter uma solução analítica. Assim, procedimentos numéricos são aplicados (Glasser, 1969; Chareonsuk et al., 1997). 


\subsection{Função utilidade multiatributo}

Em MAUT as preferências do decisor são modeladas a fim de se obter uma função utilidade multiatributo U(C,R). Esta função agrega (Vincke, 1992; Keeney \& Raiffa, 1976; Gomes et al., 2002) ambas utilidades unidimensionais $\mathrm{U}(\mathrm{C})$ e $\mathrm{U}(\mathrm{R})$. Portanto, uma função analítica é obtida para combinar ambos atributos (ou critérios) $\mathrm{C}$ e R.

Cada forma analítica de função utilidade multiatributo tem suas condições de independência em preferência avaliadas, a fim de garantir que as preferências do decisor estejam associadas com os axiomas básicos da teoria MAUT. As funções utilidade multiatributo mais usuais são a aditiva e a multilinear (Keeney \& Raiffa, 1976; Gomes et al., 2002). Estas funções são funções típicas, encontradas em vários problemas, inclusive em problemas de manutenção (Almeida \& Souza, 1993; Almeida \& Bohoris, 1995).

A função utilidade aditiva é justificada nos casos em que se encontra a condição de independência aditiva entre $\mathrm{C}$ e $\mathrm{R}$ nas preferências do decisor. A função utilidade multilinear é justificada nos casos em que se encontra a condição de independência em utilidade entre C e R (ver Keeney \& Raiffa, 1976; Gomes et al., 2002).

As funções utilidade aditiva e multilinear são respectivamente apresentadas a seguir:

$$
\begin{aligned}
& \mathrm{U}(\mathrm{C}, \mathrm{R})=\mathrm{K}_{1} \mathrm{U}(\mathrm{C})+\mathrm{K}_{2} \mathrm{U}(\mathrm{R}) \\
& \mathrm{U}(\mathrm{C}, \mathrm{R})=\mathrm{K}_{1} \mathrm{U}(\mathrm{C})+\mathrm{K}_{2} \mathrm{U}(\mathrm{R})+\mathrm{K}_{3} \mathrm{U}(\mathrm{C}) \mathrm{U}(\mathrm{R})
\end{aligned}
$$

Onde: $\mathrm{K}_{1}, \mathrm{~K}_{2}$ e $\mathrm{K}_{3}$ são constantes de escala; $\mathrm{K}_{1}+\mathrm{K}_{2}=1$ para aditiva; e $\mathrm{K}_{1}+\mathrm{K}_{2}+\mathrm{K}_{3}=1$ para multilinear.

\subsection{Função utilidade unidimensional}

Quando da obtenção da função utilidade, o comportamento do decisor em relação a risco (propensão, aversão ou neutralidade) é considerado através da função utilidade, ao contrário do procedimento no qual as variáveis são analisadas diretamente no modelo de decisão (Raiffa, 1970; Keeney \& Raiffa, 1976; Gomes et al., 2002).

Uma das mais importantes propriedades da teoria da utilidade para o caso de problemas de manutenção, está relacionada ao tratamento da incerteza, quando da análise de variáveis, tal como C e R. Uma vez que a função utilidade $U(C)$ e $U(R)$ seja obtida, então, pela teoria da utilidade, as funções utilidade finais podem ser deduzidas para qualquer que seja a função probabilística sobre C e R (Berger, 1985; Raiffa, 1970; Keeney \& Raiffa, 1976; Gomes et al., 2002).

A modelagem de preferências é aplicada ao decisor a fim de se avaliar os valores relativos das variáveis $\mathrm{C}$ e $\mathrm{R}$. Para isto há vários procedimentos de elicitação disponíveis na literatura (Raiffa, 1970; Keeney \& Raiffa, 1976). A seguir são apresentadas as funções para C e R, obtidas conforme procedimento proposto por Keeney \& Raiffa (1976).

\subsection{Função utilidade para custo}

A função utilidade exponencial é encontrada com freqüência em situações como esta (Raiffa, 1970; Keeney \& Raiffa, 1976). Em estudos prévios na área de manutenção (Almeida, 2001) 
esta função foi aplicada ao custo. Nesta função quanto maiores os valores de $\mathrm{C}$, mais indesejável se torna a alternativa para o decisor. A seguir a função:

$$
\mathrm{U}(\mathrm{C})=\mathrm{B}_{1} \mathrm{e}^{-\mathrm{A}_{1} \mathrm{C}}
$$

onde, $\mathrm{A}_{1}$ e $\mathrm{B}_{1}$ são parâmetros da função utilidade exponencial para custo.

\subsection{Função utilidade para confiabilidade}

Para confiabilidade duas funções são consideradas:

- A função utilidade linear

$$
\mathrm{U}(\mathrm{R})=\mathrm{A}_{2} \mathrm{R}+\mathrm{B}_{2}
$$

- A função utilidade logística

$$
\mathrm{U}(\mathrm{R})=\mathrm{B}_{2} \mathrm{e}^{-\frac{\mathrm{A}_{2}}{\mathrm{R}}}
$$

onde, $\mathrm{A}_{2}$ e $\mathrm{B}_{2}$ são parâmetros da função utilidade para confiabilidade. Para simplificação considerou-se a mesma notação para os parâmetros da função linear ou para logística, visto que apenas uma das alternativas será aplicada, conforme seja o resultado da modelagem de preferências com o decisor.

A função linear representa uma situação de comportamento com neutralidade a risco pelo decisor. A função logística acima apresenta uma situação de aversão a risco para valores maiores de $\mathrm{R}$ e propenso a risco para valores baixos de $\mathrm{R}$. Nas avaliações efetuadas, esta última função se ajustou melhor aos dados obtidos com os gerentes.

\subsection{Solução final}

A função $U\left(a_{i}, \theta\right)$ é derivada a partir da função utilidade $U(C, R)$, com base na propriedade de linearidade da teoria da utilidade, conforme já mencionado. Isto é, $U\left(a_{i}, \theta\right)$ é a média de $\mathrm{U}(\mathrm{C}, \mathrm{R})$, conforme (3).

Neste problema em particular, a partir de (4) e (8), observa-se que R e C são diretamente relacionados com $\theta$ (parâmetros associados com $\mathrm{R}$ ) e com a alternativa $\mathrm{a}_{\mathrm{i}}$ (associada com $\mathrm{t}_{\mathrm{p}}$ ). Então, $\mathrm{U}\left(\mathrm{a}_{\mathrm{i}}, \theta\right)=\mathrm{U}(\mathrm{C}, \mathrm{R})$ dado (4) e (8). Isto é:

$$
\mathrm{U}\left(\mathrm{a}_{\mathrm{i}}, \theta\right)=\mathrm{U}(\mathrm{C}, \mathrm{R}) \text { Tal que } \mathrm{C} \text { seja dado por (8) e R seja dado por (4) }
$$

Assim, o valor esperado de $\mathrm{U}\left(\mathrm{a}_{\mathrm{i}}, \theta\right)$ em (1) torna-se:

$$
\begin{aligned}
\mathrm{E}_{\theta}\left\{\mathrm{U}\left(\mathrm{a}_{\mathrm{i}}, \theta\right)\right\}= & \int_{\theta}\{\mathrm{U}(\mathrm{C}, \mathrm{R}) \pi(\theta)\} \mathrm{d} \theta \\
& \text { tal que C seja dado por (8) e R seja dado por (4). }
\end{aligned}
$$


Conforme previamente mencionado, há duas situações relativas ao parâmetro $\theta$ :

- Os parâmetros de $\theta$ são conhecidos, e

- Os parâmetros de $\theta$ não são conhecidos, mas há uma probabilidade a priori $\pi(\theta)$ obtida de um especialista, permitindo a adoção da abordagem Bayesiana.

Para o primeiro caso, a alternativa ótima é obtida para a máxima utilidade $\mathrm{U}\left(\mathrm{a}_{\mathrm{i}}, \theta\right)$ em $(14)$.

Para o segundo caso, a alternativa ótima é obtida aplicando (15) sobre (2). Assim, a alternativa ótima é obtida para a máxima utilidade esperada $\mathrm{E}_{\theta} \mathrm{U}\left(\mathrm{a}_{\mathbf{i}}, \theta\right)$.

Para ambos os casos, com respeito ao custo, (8) é aplicado sobre (11), o qual sobre a função utilidade multiatributo $\mathrm{U}(\mathrm{C}, \mathrm{R})$. Dependendo do resultado da modelagem em preferências esta função $U(C, R)$ pode ser representada por (9) ou (10).

Similarmente, a função confiabilidade em (4) é aplicada sobre a função utilidade para a confiabilidade $U(R)$. A função utilidade $U(R)$ pode ser (12) ou (13), de acordo com a forma analítica obtida para $\mathrm{U}(\mathrm{R})$, como resultado da modelagem de preferências junto ao decisor. Finalmente aplica-se este resultado de (4) em $\mathrm{U}(\mathrm{R})$ sobre a função utilidade multiatributo $\mathrm{U}(\mathrm{C}, \mathrm{R})$. Conforme já descrito acima, U(C,R) pode ser representada por (9) ou (10), de acordo com a forma selecionada para a função $U(C, R)$.

\section{Aplicação Numérica}

Com o objetivo de ilustrar o uso do modelo, uma aplicação numérica é apresentada. Esta aplicação é baseada em um estudo real em uma empresa de energia elétrica, embora partes do estudo não apresentem os mesmos resultados numéricos, por questão de restrição a divulgação. Neste contexto, tem-se um sistema de produção de serviços, onde as conseqüências não são completamente visualizadas através de uma análise de custos. A confiabilidade do sistema deve completar a visão multiobjetivo para este tipo de sistema.

O sistema de produção em uma empresa de energia elétrica se caracteriza no contexto de um sistema de serviços. Conforme justificado neste tipo de sistema a abordagem multicritério torna-se muito importante. Neste sistema o usuário de energia elétrica está em direto contato direto com o sistema de produção. O produto energia suprida é produzido enquanto o cliente está sendo servido. Assim, a energia recebida pelo usuário é diretamente afetada com os distúrbios decorrentes de falhas no sistema de energia elétrica. As perdas decorrentes de interrupções acarretam distúrbios imediatos no usuário. Esta situação é crítica quando se consideram hospitais e outros serviços públicos, ou mesmo na interrupção de usuários industriais. Neste caso, as conseqüências de falhas não podem facilmente ser transformadas em custos. Assim, a confiabilidade do sistema é uma conseqüência de desempenho que pode ser avaliada neste modelo multicritério que associa também o custo.

Os seguintes dados e condições foram obtidos do sistema e do contexto estudado:

- Os custos são dados por $\mathrm{C}_{\mathrm{f}}=1500$ e $\mathrm{C}_{\mathrm{p}}=700$ em unidades monetárias. Estes parâmetros são aplicados em (8).

- O espaço de ações foi definido por intervalos de tempo múltiplos de 60 , correspondendo a 60 dias, para cada intervalo. Assim, tem-se que $a_{1}=60 ; a_{2}=120 ; a_{3}=180$; isto é $\mathrm{a}_{\mathrm{i}}=60 \mathrm{i}, \mathrm{i}=1,2,3, \ldots$ 
- A partir da análise de dados de falhas do sistema, para a função probabilidade relativa a $\mathrm{f}(\mathrm{t})$ foi obtida a função Weibull, com parâmetros: $\beta=3,5$ e $\eta=1500$. Esta função é aplicada em (4) e (8).

- A partir da elicitação da função utilidade multiatributo, foi confirmada a função utilidade aditiva com parâmetros $\mathrm{K}_{1}=0,6$ e $\mathrm{K}_{2}=0,4$, aplicados em (9).

- A partir da elicitação da função utilidade para $\mathrm{U}(\mathrm{C})$, foram obtidos os parâmetros para (11): $\mathrm{A}_{1}=0,7$ e $\mathrm{B}_{1}=1,35$.

- A partir da elicitação da função utilidade para $U(R)$, foi confirmada a função utilidade logística e foram obtidos os parâmetros para (13): $\mathrm{A}_{2}=2,1$ e $\mathrm{B}_{2}=8,2$.

A utilidade para cada alternativa é encontrada aplicando-se (14). A Figura 1 mostra as utilidades obtidas para cada alternativa $a_{i}$. A alternativa ótima foi encontrada para $i=9$, indicando a9 $=540$ dias. Uma análise de sensibilidade mostra que a alternativa recomendada permanece a mesma com uma variação de todos os parâmetros em torno de mais ou menos $20 \%$, exceto dos parâmetros $\mathrm{K}_{1}$ e $\mathrm{K}_{2}$, que apresentam a mesma recomendação de ay, apenas quando variam em torno de mais ou menos $10 \%$.

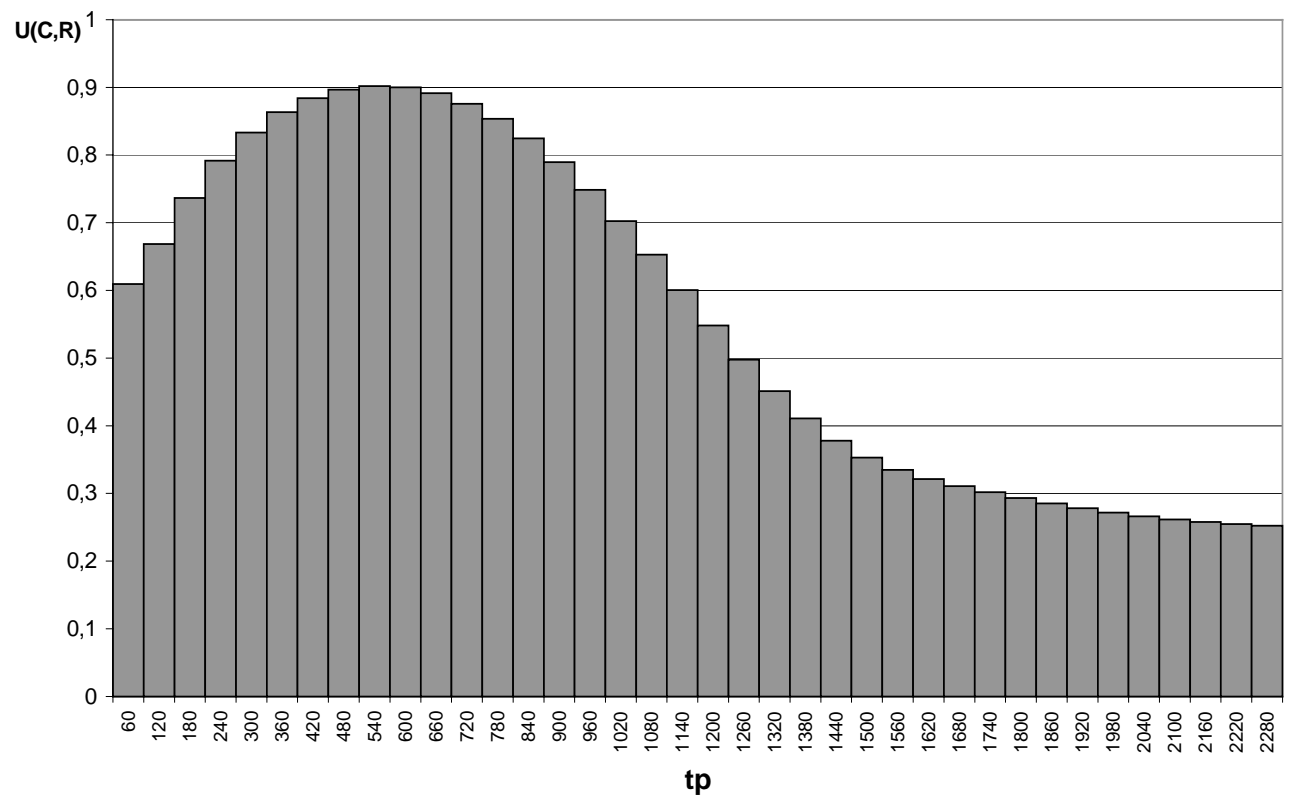

Figura 1 - Utilidade de $a_{i}-U\left(a_{i}, \theta\right)$ versus alternativa $a_{i}\left(t_{p}\right)$.

A Figura 2 mostra a função custo em função do tempo para intervenção preventiva $\left(t_{p}\right)$, dada pela equação (8), donde se observa a solução pela abordagem baseada apenas no custo, correspondente ao modelo clássico. 


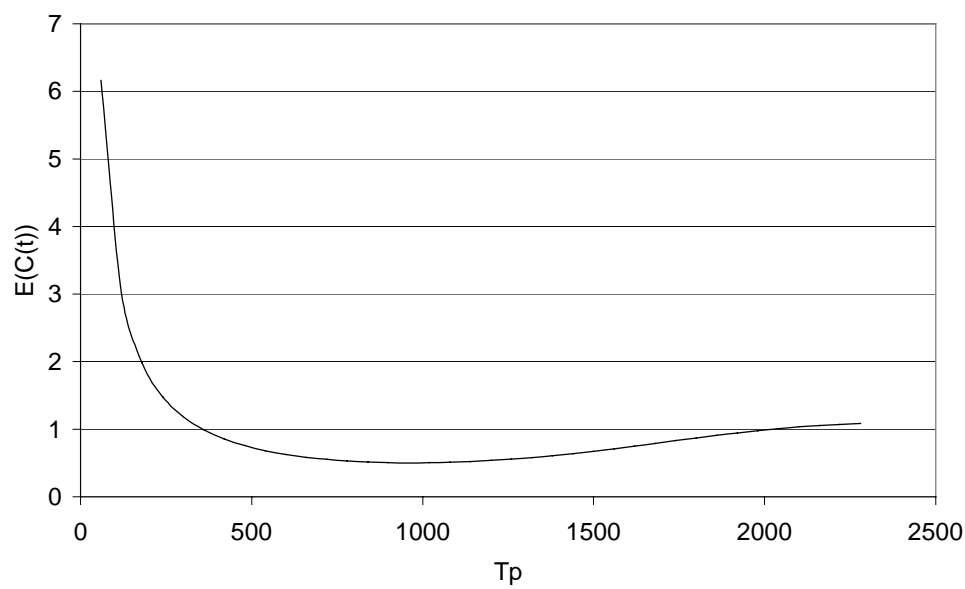

Figura 2 - Custo esperado por tp.

Aplicando-se o modelo clássico, considerando apenas o custo esperado, conforme a Figura 2, a alternativa ótima é encontrada para $\mathrm{i}=16$, isto é, $\mathrm{a}_{16}=960$. No entanto, a melhor alternativa para o decisor ocorre para $t_{p}=540$, aplicado-se o modelo aqui proposto. Pode-se observar que esta alternativa apresenta um custo mais alto, que é compensado pela maior confiabilidade, de acordo com a própria função utilidade multiatributo do decisor.

A Figura 3 mostra a função utilidade para o custo esperado.

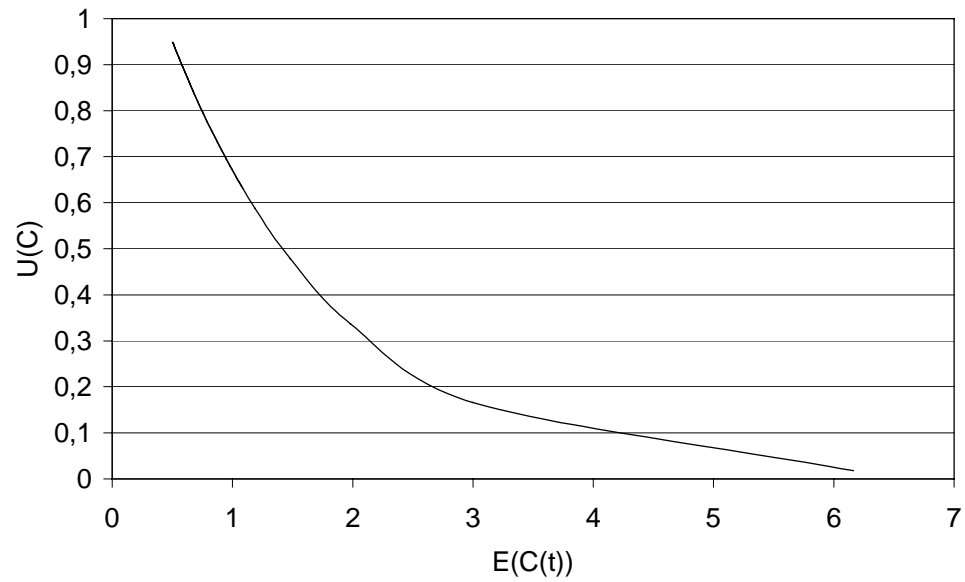

Figura 3 - Utilidade U(C).

A Figura 4 mostra a confiabilidade do sistema em função do tempo de intervenção t. Conforme se observa a confiabilidade para tp $=960$ está em torno de 0,8 . Já para alternativa selecionada através do modelo de decisão multicritério, $\mathrm{t}_{\mathrm{p}}=540$, a confiabilidade está em torno de 0,97. 


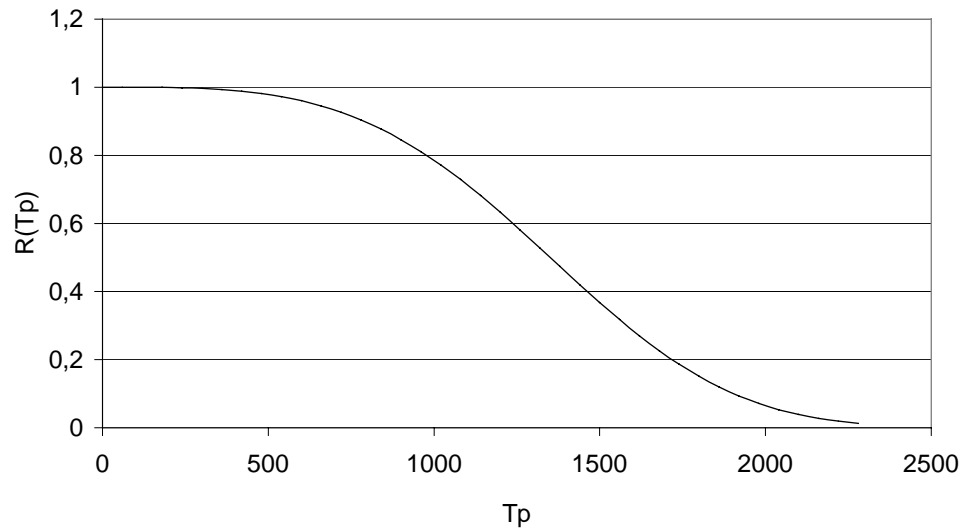

Figura 4 - Função confiabilidade R(tp).

A função utilidade para a confiabilidade é apresentada na Figura 5, para a qual observa-se que está limitada a $\mathrm{R}=1$, com utilidade $\mathrm{U}(\mathrm{R})=1$. Conforme se observa, a utilidade no atributo confiabilidade para a solução pelo método sem abordagem multicritério (onde $\mathrm{R}=0,8)$ está em torno de 0,6 .

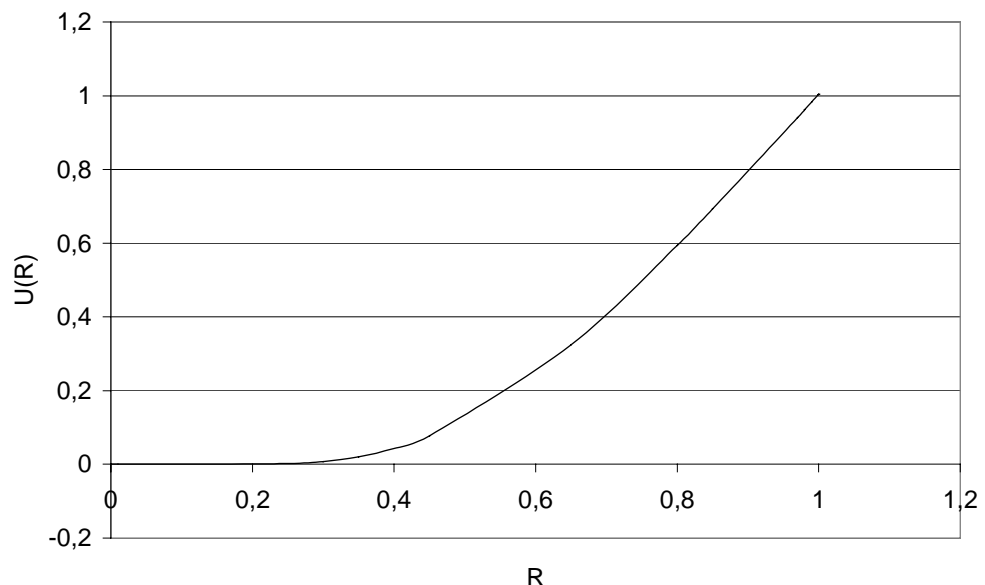

Figura 5 - Utilidade para a confiabilidade U(R).

Considerando as preferências do decisor, expressas através da função utilidade apresentada, tem-se que a confiabilidade influencia o estabelecimento do tempo de intervenção para manutenção preventiva, de modo a reduzir a periodicidade em comparação com a abordagem baseada apenas na minimização dos custos. 


\section{Conclusões}

O problema de determinação de periodicidade para intervenção de manutenção preventiva foi analisado considerando a abordagem MAUT. É importante destacar que os problemas de decisão na área de manutenção são tipicamente problemas sob situação de incerteza, requerendo a metodologia apropriada. Na maioria dos casos MAUT atende bem a esta tarefa.

Embora para sistemas de produção de bens, uma modelagem monocritério possa ser adequada, para sistemas de produção de serviços isto não é muito apropriado. Nestes contextos, uma abordagem multicritério é importante por permitir a incorporação da análise dos impactos ocasionados ao cliente pela interrupção dos serviços.

Foram empregados dois critérios para analisar este problema: custo e confiabilidade. As abordagens mais clássicas utilizam apenas o critério custo, encontrando alternativa para o qual o custo é minimizado. Foi mostrado o impacto da introdução do critério confiabilidade no modelo de decisão, recomendando uma intervalo menor para intervenção preventiva, de modo a atender ao cliente com uma confiabilidade maior, embora compensado com certo aumento nos custos, em quantidade compatível com as preferências do decisor, expressas na função utilidade.

\section{Agradecimentos}

Este trabalho foi parcialmente apoiado pelo CNPq.

\section{Referências Bibliográficas}

(1) Almeida, A.T. de \& Bohoris, G.A. (1995). Decision Theory in Maintenance Decision Making. Journal of Quality in Maintenance Engineering, 1(1), 39-45.

(2) Almeida, A.T. de \& Souza, F.M.C. (1993). Decision Theory in Maintenance Strategy for a Two-Unit Redundant Standby System. IEEE Transaction on Reliability, 42(3), 401-407.

(3) Almeida, A.T. de (2001). Multicriteria Decision Making on Maintenance: Spares and Contracts Planning. European Journal of Operational Research, 129(2), 235-241.

(4) Barlow, R.E. \& Hunter, L. (1960). Optimum Preventive Maintenance Polices. Operations Research, 8(1), 90-100.

(5) Berger, J.O. (1985). Statistical Decision Theory and Bayesian Analysis. SpringerVerlag, Berlin.

(6) Chareonsuk, C.; Nagarur, N. \& Tabucanom, M.T. (1997). A Multicriteria Approach to the Selection of Preventive Maintenance Intervals. International Journal of Production Economics, 49(1), 55-64.

(7) Dekker, R. \& Scarf, P.A. (1998). On the Impact of Optimisation Models in Maintenance Decision Making: The State of the Art. Reliability Engineering \& System Safety, 60(2), 111-119.

(8) Glasser, G.J. (1969). Planned Replacement: Some Theory and its Application. Journal of Quality Technology, 1(2), 110-119. 
(9) Gopalaswamy, V.; Rice, J.A. \& Miller, F.G. (1993). Transit Vehicle Component Maintenance Policy via Multiple Criteria Decision Making Methods. The Journal of the Operational Research Society, 44(1), 37-50.

(10) Gomes, L.F.A.M.; Gomes, C.F.S. \& Almeida, A.T. (2002). Tomada de Decisão Gerencial: Enfoque Multicritério. Editora Atlas, Rio de Janeiro.

(11) Keeney, R.L. \& Raiffa, H. (1976). Decision with Multiple Objectives: Preferences and Value Trade-offs. John Wiley \& Sons, New York.

(12) Kralja, B. \& Petrovicb, R. (1995). A Multiobjective Optimization Approach to Thermal Generating Units Maintenance Scheduling. European Journal of Operational Research, 84(2), 481-493.

(13) Martz, H.F. \& Waller, R.A. (1982). Bayesian Reliability Analysis. John Wiley \& Sons, New York.

(14) O’Connor, P.D.T. (1985). Practical Reliability Engineering. John Wiley \& Sons, New York.

(15) Percy, D.F. \& Kobbacy, K.A.H. (2000). Determining Economical Maintenance Intervals. International Journal of Production Economics, 67(1), 87-94.

(16) Raiffa, H. (1970). Decision Analysis. Addison-Wesley.

(17) Vincke, P. (1992). Multicriteria Decision-Aid. John Wiley \& Sons. 